\title{
Single-Study Approvals: Quantum of Evidence Required
}

Therapeutic Innovation \& Regulatory Science 2020, Vol. 54(3) 687-692 (C) The Author(s) 2020 https://doi.org//0.1007/s43441-019-00103-4

\author{
Frank J. Sasinowski, MS, MPH, JD', and Michelle L. Butler, JD' $\odot$
}

\begin{abstract}
When does a single positive adequate and well-controlled study of a new drug meet the statutory requirement to demonstrate substantial evidence of effectiveness? The answer to this question, particularly with respect to new molecular entities, has been of considerable debate since 1962 when the requirement that new drugs prove their benefit to patients became law. A 1997 revision to the statute provided one pathway to a single-study approval (a single adequate and well-controlled study plus confirmatory evidence), while a 1998 guidance issued by FDA provided additional pathways, one of which is the one that is most frequently cited by FDA (a single statistically very persuasive study). This paper explains these 2 distinct pathways and provides illustrative examples of how FDA uses each of these 2 pathways. Regulators, industry, patients, and investors should each find this exegesis of these 2 independent, yet equally viable and valuable, pathways to an FDA approval both illuminating and invaluable.
\end{abstract}

\section{Keywords}

FDAMA I I5, confirmatory evidence, substantial evidence, evidence guidance, effectiveness

\section{Introduction}

The question of what quantum of evidence constitutes "substantial evidence of effectiveness" for a drug that is a new molecular entity (NME) has been debated since 1962 when the requirement to demonstrate the effectiveness of a drug became law. In some cases, a single adequate and well-controlled pivotal study may provide such substantial evidence, either alone or in conjunction with other information. Between the Federal Food, Drug, and Cosmetic Act (FDC Act) and guidance issued by the Food and Drug Administration (FDA or the Agency), there are 2 distinct pathways in which a single study may provide the quantum of effectiveness evidence required for approval of a drug. One of these is described in FDA guidance: a single adequate and well-controlled study with certain characteristics including and especially "a statistically very persuasive finding" (hereinafter a "single statistically very persuasive study"). The other comes from section 115 of the Food and Drug Administration Modernization Act (FDAMA 115): a normal or conventional adequate and well-controlled study plus "confirmatory evidence" (hereinafter a "single study with confirmatory evidence"). Since these 2 pathways came to be approximately 2 decades ago, drug companies (sponsors) and even FDA have generally focused on the former pathway: a single statistically very persuasive study. Yet, our review of FDA drug approvals as precedents shows that FDA employs both pathways for approving NMEs, and sponsors are therefore able to rely on either pathway in planning an NME's clinical program.

\section{Legal and Regulatory Background and Exegesis of the Two Pathways}

In 1962, the FDC Act was amended to require a demonstration of substantial evidence of efficacy in order for a drug to be approved. ${ }^{i}$ Specifically, the 1962 amendment defined "substantial evidence" as "adequate and well-controlled investigations, including clinical investigations, ... to evaluate the effectiveness of the drug involved, on the basis of which it could fairly and responsibly be concluded ... that the drug will have the effect it purports or is represented to have....", FDA has generally interpreted this quantum of evidence as at least 2 adequate and well-controlled studies, each of which provides evidence of the investigational therapy's benefit to patients. ${ }^{1}$

In March 1997, FDA published a draft guidance document, "Providing Clinical Evidence of Effectiveness for Human Drug and Biological Products," which stated that even though substantial evidence is generally considered to be at least 2 adequate and well-controlled studies, "nevertheless, FDA has been flexible within the limits imposed by the Congressional scheme, broadly interpreting the statutory requirements to the

\footnotetext{
' Hyman, Phelps \& McNamara, PC, Washington, DC, USA

Submitted 03-Dec-2018; accepted 08-Mar-2019; published online 6-Jan-2020 Corresponding Author:

Michelle L. Butler, JD, Hyman, Phelps \& McNamara, PC, 700 13th St NW, Washington, DC 20005, USA.

Email: MButler@hpm.com
} 
extent possible where the data on a particular drug are strong."2 The draft guidance document stated that "in some cases FDA has relied on only a single adequate and well-controlled efficacy study to support approval - generally only in cases in which a single multicenter study of excellent design provided highly reliable and statistically strong evidence of an important clinical benefit, such as an effect on survival, and a confirmatory study would have been difficult to conduct on ethical grounds." ${ }^{2}$ This document then provided prospective guidance on "the quantity of evidence needed in particular circumstances to establish substantial evidence of effectiveness, "iii including situations in which a single statistically very persuasive study would be appropriate. While guidance documents do not contain legal requirements, they do provide insight into the Agency's thinking at the time. This draft guidance document demonstrated that FDA was willing to consider, in appropriate circumstances, a single adequate and well-controlled study as sufficient for approval under the 1962 statutory effectiveness standard.

In November 1997, FDAMA 115 amended the FDC Act by creating a second, equally valid, alternate way in which to demonstrate the required statutory standard of substantial evidence of effectiveness needed for approval:

If [FDA] determines, based on relevant science, that data from one adequate and well-controlled clinical investigation and confirmatory evidence (obtained prior to or after such investigation) are sufficient to establish effectiveness, [FDA] may consider such data and evidence to constitute substantial evidence. ${ }^{\text {iv }}$

In May 1998, FDA finalized its effectiveness guidance document, with the substance of the guidance nearly unchanged from the draft version. ${ }^{\mathrm{v}}$ While this guidance discusses multiple situations in which a single study may support an effectiveness determination, this paper focuses on one of those situations that has been the most often and widely cited by FDA when it refers to a "single-study approval" standard, particularly in the context of an NME. ${ }^{\mathrm{vi}}$

FDA, in its guidance, "identifies the characteristics of a single adequate and well-controlled study that could make the study adequate" for demonstrating substantial evidence of effectiveness. ${ }^{\text {vii }}$ FDA lists several characteristics, such as whether that single study was a large multicenter study in which no single study site provided an unusually large fraction of the patients or is disproportionately responsible for the favorable effect seen. Other factors that FDA lists are similarly there to illustrate the kinds of characteristics that FDA would consider as strengthening that single study's showing of benefit rather than to compel that each and every characteristic be present. Such characteristics include consistency across study subsets, multiple studies in a single study, and multiple prospectively identified primary or secondary endpoints, each of which represents a beneficial, but different, effect. ${ }^{\text {viii }}$ The only characteristic listed by FDA in its guidance that in practice FDA has elevated to being absolutely necessary (but even this is not on its own sufficient) is that the results of this single study achieve "a statistically very persuasive finding." fore, this paper's "shorthand" for this pathway is a "single statistically very persuasive study."

Did this mean that FDA (with its 1997/1998 Evidence Guidance announcing a "single statistically very persuasive study" standard) as well as Congress and the President (with the FDAMA 115 "single study with confirmatory evidence" standard) at roughly the same time were "watering down" the requirements for a new medicine to be approved? Hardly. It was simply that each was understanding, based on the experience of the intervening $3 \frac{1}{2}$ decades since the 1962 law had created the requirement to demonstrate effectiveness, that sometimes the conventional 2 positive trials requirement could be met in other ways.

In the case of FDA's guidance, the 2-trial effectiveness requirement could essentially be satisfied in reliance on "a statistically very persuasive finding" and other characteristics of that single study that would provide a comparably reliable and credible level of evidence in that single trial as may otherwise be derived from 2 trials, each with a $P$ value less than .05. Similarly, under FDAMA 115, "confirmatory evidence" could serve to stand in or substitute for the second positive adequate and well-controlled trial. One may think of these 2 pathways as situations in which there exists a second trial but one that is nested within the single trial in the case of the FDA guidance or one that is a "phantom" trial in the case of FDAMA 115.

What is noteworthy is that there was a particularly propitious moment in time 20 years ago when FDA and Congress each recognized the public health good that could flow from specifying a single-study approval pathway as an alternative to the oft-stated proposition that the 1962 law that first required a showing of benefit did so in terms interpreted to mean that a new medicine had to prove that it benefited patients in a minimum of 2 positive hypothesis-testing trials.

\section{Pathway I: Single Statistically Very Persuasive Study-Precedents}

This paper starts with illustrative precedents of this type of single-study approval because this is the pathway that historically has dominated most discussions of the single-study approval approach. ${ }^{\mathrm{xi}}$

A recent example is Giapreza ${ }^{\mathrm{TM}}$ (angiotensin II) injection for intravenous infusion, which was approved in December 2017 to increase blood pressure in adults with septic or other distributive shock. ${ }^{3, x i i}$ In this case, "the demonstration of benefit is based on a single, multinational, randomized, placebocontrolled trial in 344 adults with distributive shock and persistent hypotension." 4 The primary endpoint assessed the proportions of "responders" at 3 hours. Response rates were $70 \%$ in the angiotensin II group (114 of 163 subjects) and $23 \%$ in the placebo group $(37$ of 158 subjects $)(P<.0001) .{ }^{4}$ This one trial provides the equivalent of 2 positive trials that are each 
just slightly less than a $P$ value of .05 , either to a trialist or on an evidentiary spectrum; it is as if a second trial were nested within this single trial. According to the FDA Office Director's Memorandum:

As noted [in the Cross-Discipline Team Leader Review], the study has many of the characteristics of a single adequate and wellcontrolled study that could, by itself, provide evidence of effectiveness, as outlined in FDA's 1998 Guidance for Industry: "Providing Clinical Evidence of Effectiveness for Human Drugs and Biological Products." The effect size (47\% absolute increase) is large and clinically meaningful on its face. The results are statistically persuasive, and robust to any type of exploration. Positive results were observed across sites in the U.S., Canada, and Australia, and across other important baseline demographic and disease subsets. The population studied seems generalizable to the US population at large. ${ }^{4}$

A second recent example of a single-study approval of this type is Bevyxxa ${ }^{\circledR}$ (betrixaban), which was approved in June 2017 for the prophylaxis of venous thromboembolism (VTE) in adult patients hospitalized for an acute medical illness who are at risk for thromboembolic complications because of moderate or severe restricted mobility and other risk factors for VTE. In this case, the sponsor submitted the data from a single randomized, double-blind, multinational study that randomized 7513 patients and compared extended duration of oral betrixaban to short duration of injectable enoxaparin in the prevention of VTE. According to FDA's review of the data, the study failed to reach statistical significance on the prespecified primary analysis of the primary endpoint, which was cohort 1 of the primary efficacy outcome population (PEOP) (the relative risk in the betrixaban arm relative to the enoxaparin arm was $80.6 \%$, with $95 \%$ confidence interval [CI] of $64.7 \%, 100.4 \%$, $P=.054) .^{5}$ As a result, FDA viewed all other analyses as exploratory and no other $P$ values are included in the review documents or the betrixaban labeling. However, according to a published report of the study's findings, cohort 1 yielded a $P$ value of .048 , ${ }^{6, x i i i}$ with even more impressively robust results seen in cohort 2 of the PEOP and the Overall PEOP (in cohort 2 , the relative risk in the betrixaban arm relative to the enoxaparin arm was $79.6 \%$, with $95 \%$ CI of $65.2 \%, 97.3 \%$, $P=.025$; in Overall PEOP, the relative risk in the betrixaban arm relative to the enoxaparin arm was $75.7 \%$, with $95 \%$ CI of $62.3 \%, 91.9, P=.005)$. ${ }^{\text {xiv }}$

While the review documents do not mention the 1998 Evidence Guidance, it is apparent that it was the basis for approval as the review documents make no mention of any confirmatory or related study data. It is even more telling that the betrixaban study meets several of the characteristics identified in the 1998 Evidence Guidance: a large multicenter study, consistency across subsets, consistency across components of the primary efficacy endpoint, and a $P$ value of less than .01 in the Overall PEOP analysis.
A third recent example is Spinraza ${ }^{\mathrm{TM}}$ (nusinersen), which was approved in December 2016 for the treatment of spinal muscular atrophy (SMA) in pediatric and adult patients. In this case, the sponsor submitted data from a planned interim analysis of a double-blind, sham-controlled trial in subjects with infantile-onset SMA who had 2 copies of the SMN2 gene (Study CS3B). The primary endpoint was a responder analysis based on achievement of motor milestones. "The trial demonstrated a clear and important benefit of nusinersen, with 21/51 $(41 \%)$ of nusinersen-treated patients meeting [the] responder definition ...vv. 0/27 $(0 \%)$ of controls $(p<0.0001)$." ${ }^{, 7}$ In addition, the secondary endpoints, although presented only descriptively, consistently supported a treatment benefit.

According to the FDA Office Director's Memorandum, "the study has many of the important characteristics of an adequate and well-controlled study that can, by itself, provide substantial evidence of effectiveness, as outlined in FDA's [1998 Evidence Guidance] (see pages 15-16)., ${ }^{, 7, x v}$ In coming to the conclusion that the study provides substantial evidence of effectiveness, the memorandum considered whether it would be ethical to conduct a second study in which some patients would be randomized to a sham procedure, determining that such a study would not be ethical or practicable. ${ }^{7(p 15)}$

This same memorandum then stated that FDA "typically look[s] for confirmatory evidence of effectiveness when considering approval based on a single adequate and well-controlled trial. ${ }^{7(\mathrm{p} 15)}$ The memorandum concluded that additional studies, such as an open-label study in infantileonset SMA (Study CS3A) and a controlled trial in patients with later-onset SMA (Study CS4), the high-level results of which were submitted during the review cycle, "constitute the "confirmatory evidence' that would be needed for an approval action based on a single adequate and well-controlled trial, which is also supported by the statistical strength of the Study CS3B result." ${ }^{\text {, (p16) }}$ The range of ages of disease onset covered by the studies permitted the efficacy findings to be generalized to adult-onset SMA.

Note how this approval illustrates BOTH types of singlestudy approval pathways. Initially, FDA lays out the basis for an approval based on the statistically very persuasive finding in study CS3B, including direct FDA reference to the 1998 Effectiveness Guidance and one of the characteristics that FDA may consider in such an action, which is that a second trial in infantile-onset SMA may be unethical given the robust results in study CS3B. Then, in the same decisional memorandum, FDA pivots and explains that in addition to having evidence sufficient to find a basis for approval on this single statistically persuasive study on its own, FDA finds that there is confirmatory evidence also, which would have permitted or led FDA to an approval based on this second pathway too. Kudos to FDA for understanding and explaining to sponsors, patients, congressional oversight, and investors how FDA may have used either one of these 2 pathways to have approved this therapy.

These are but 3 of the more recent examples from many more precedents that each show FDA's willingness to approve 
NMEs on the basis of a single statistically very persuasive adequate and well-controlled trial, standing on its own without any other supportive or confirmatory evidence.

\section{Pathway 2: Single Study with Confirmatory Evidence-Precedents}

As mentioned above, the 1998 Evidence Guidance does not define or otherwise provide insights on what would be considered "confirmatory evidence" sufficient to support a single clinical study that might not have the characteristics discussed above for a pathway 1 approval.

A seminal example of an approval based on pathway $2-a$ single study together with confirmatory evidence-is the approval of the NME, iloprost, for primary pulmonary hypertension. In his 2004 memorandum explaining the decision to approve this drug, Dr Robert Temple, Director of Office of Drug Evaluation I at the time, stated that in addition to the single positive trial, evidence from related therapy can be the "confirmatory evidence" needed under FDAMA $115 .{ }^{8}$ And what did Dr Temple point to as the confirmatory evidence sufficient to support this single trial? He found that "the strongest external support comes from the closely related prostacyclin analogues epoprostenol... and treprostinil." 8 In plain terms, the confirmatory evidence needed to support the approval of iloprost was that iloprost was pharmacologically closely related to the only 2 other drugs FDA had approved for this condition. This pharmacological class nexus in effect served as the phantom second trial or confirmatory evidence. What makes this even more revealing of the regulatory nimbleness of FDA is that both of these 2 prior FDA approvals were injectables, while iloprost was an inhalation therapy.

Dr Temple in that same memo also pointed to 2 prior additional examples of the use of confirmatory evidence. First, he cited to the approval of 2 angiotensin II blockers (irbesartan and losartan) that had supplied the confirmatory evidence for each other in delaying functional deterioration in type II diabetes. Here too it was the pharmacological connectedness or relatedness of the 2 different molecules that provided the confirmatory evidence for the other. Second, Dr Temple referenced another instance in which a well-established pharmacologic class effect was the confirmatory evidence when he explained that angiotensin-converting enzyme inhibitors had been approved for congestive heart failure based on single studies "with $\mathrm{p}$ values between 0.05 and 0.01 with the backdrop of multiple drugs in the class showing favorable effect." ${ }^{\text {xvi }}$

In 2002, in the only known workshop on FDAMA 115 , Dr Janet Woodcock, director of the FDA Center for Drug Evaluation and Research, and one of the coauthors of this article co-chaired the key breakout session intended to define what is meant by "confirmatory evidence." 9 In this breakout session, Dr Woodcock pointed to the ability of FDA to rely on "prior scientific knowledge" of a pharmacologic class as confirmatory evidence to support a single-study approval. In the breakout session, Dr Woodcock and one of this paper's coauthors discussed the nature and limits of what may constitute confirmatory evidence. During the discussion, the coauthor asked whether, for an estrogen that was an NME intended for treating postmenopausal symptoms that had a single study with a $P$ value between .05 and .01 , the confirmatory evidence could simply be all the prior literature and approvals for other estrogens to treat this same indication of postmenopausal symptoms. Dr Woodcock replied that such prior scientific evidence could be the confirmatory evidence to support this single study of an NME of estrogen for the same indication.

More recently, in the case of Spinraza (nusinersen), as discussed above, FDA found open-label studies and a controlled study (for which the results were submitted during the review and therefore not independently verified by FDA) to constitute the confirmatory evidence sufficient for approval.

As with pathway 1, there are many more precedents that could be cited, but those above as well as the FDA statements, including those of Drs Woodcock and Temple, eloquently manifest FDA's willingness to embrace various types of "confirmatory evidence" to support a single-study approval based on confirmatory evidence for an NME under FDAMA 115.

\section{Conclusion}

FDA's own guidance and precedents demonstrate the Agency's commitment to be flexible in its review of certain applications for NMEs based on a single positive registration quality study. The pathway that historically has received the most attention is where there is a single study that has a statistically very persuasive finding, yet FDA has similarly embraced the FDAMA 115 "confirmatory evidence" pathway as well. FDA precedents show the viability and vitality of both pathways within the Agency, and sponsors may explore with FDA such pathways when considering whether an existing quantum of efficacy evidence is adequate for FDA to enable patients to access an NME as an FDA-approved therapy to address the needs of those patients for that medicine.

\section{Declaration of Conflicting Interests}

Both authors are employed by Hyman, Phelps \& McNamara, P.C., a law firm that represents sponsors who may be pursuing single-study approvals of their product candidates.

\section{Funding}

No financial support of the research, authorship, and/or publication of this article was declared.

\section{ORCID iD}

Michelle L. Butler, JD (D) https://orcid.org/0000-0002-5898-321X

\section{Notes}

i. See 21 USC $\S \S 355(d)(5), 355(c)(1)(A)$.

ii. Ibid, $\S 355(d)$. 
iii. 1997 Evidence Guidance, ${ }^{2}$ at 5 .

iv. 21 USC $§ 355(\mathrm{~d})$ (emphasis added).

v. 1998 Evidence Guidance, ${ }^{1}$ at 6. Although FDAMA 115 was enacted prior to FDA finalizing and issuing the 1998 Evidence Guidance, FDA did not revise its draft guidance to either define or provide its views of what might constitute "confirmatory evidence" (and no FDA guidance has yet been issued to aid sponsors in understanding the nature, function, and limits of that key phrase "confirmatory evidence" in the 1997 law).

vi. Although not a focus of this paper, the guidance also discusses when (1) extrapolation from existing studies may be sufficient to demonstrate effectiveness of a new indication of an approved product and (2) a single study "of a new use, with independent substantiation from related study data" (ibid, 7-12).

FDA in this guidance explains further that, because "[a] conclusion based on two persuasive studies will always be more secure than a conclusion based on a single, comparably persuasive study," "reliance on only a single study will generally be limited to situations in which a trial has demonstrated a clinically meaningful effect on mortality, irreversible morbidity, or prevention of a disease with potentially serious outcome and confirmation of the result in a second trial would be practically or ethically impossible" (ibid, 13). The use of the qualifier "generally" means that impossibility of a second trial is not a requirement for reliance on a single statistically very persuasive study for approval, even though that may sometimes be FDA's preference.

vii. Ibid. This pathway is meant to apply to situations in which there is reliance solely upon the single study without any other supportive or related effectiveness information from any other source. Therefore, when FDA or sponsors refer to a "totality of evidence," they are generally referring to the other pathway under FDAMA 115.

viii. According to the guidance, it is also of critical importance to consider the possibility of an incorrect outcome and examine all available data for their potential to support or undermine reliance on a single study (ibid, 15).

ix. Ibid, 13-15.

$\mathrm{x}$. One might argue that the only pathway for a single-study approval is a single study with confirmatory evidence as that is the only single study pathway identified in the FDC Act. However, before that pathway existed, FDA articulated situations in which a single-study approval may be appropriate. While guidance documents do not contain legal requirements, they do represent the Agency's thinking at the time, and the Agency was comfortable approving drugs based on the types of evidence identified in the guidance, particularly based on a single study with a statistically very persuasive finding. As the guidance was finalized after the enactment of FDAMA 115 with the substance remaining unchanged, it is clear that the Agency continues to support the single-study approval pathway articulated in the guidance, which is borne out by decisions the Agency has made to approve drugs in the intervening years, including the examples discussed under pathway 1. xi. Ibid, 13-15.

xii. This drug was approved on December 21, 2017, as an NME. Another form of angiotensin II was approved in 1961 and withdrawn in 2009. FDA determined that there was no reason to believe the withdrawal was due to safety issues. The current angiotensin II differs from the previously approved angiotensin II in that the amino acid sequence differs at the first and fifth positions. The sponsor of Giapreza submitted the application as a 505(b)(2) NDA based in part on literature, but FDA determined that "the clinical studies conducted by or for the applicant alone support the clinical safety and effectiveness of the product and support the approval of the NDA.",3 Accordingly, the sponsor submitted a revised Form 356h that identified the NDA as a 505(b)(1) NDA. ${ }^{3}$ The review also hypothesized that "[o]ne might make the argument that efficacy is supported by the efficacy of the previously marketed, closely related bovine angiotensin product, but the strength of the results of [the single trial submitted in the NDA] makes that unnecessary."3(p22)

xiii. There has been some discussion regarding a particular patient, known as Patient X, and whether that patient had a reportable event. If the patient, who was in the enoxaparin arm, is considered to have had an event, the results on the primary analysis of the primary endpoint would be statistically significant (the relative risk in the betrixaban arm relative to the enoxaparin arm was $80.2 \%$, with $95 \%$ confidence interval of $64.4 \%, 99$. $8 \% ; P=.048)$.

xiv. Gibson et al. ${ }^{6}$ These results include Patient $X$ as an event. If Patient $\mathrm{X}$ is not considered to have had a reportable event, the $P$ values for cohort 2 and Overall PEOP are $P=.029$ and $P=.006$, respectively.

xv. Topline results of a second study, a double-blind, shamcontrolled trial in patients with later-onset SMA, were submitted during review of the NDA. The sponsor reported a highly statistically significant $(P<.001)$ treatment effect as well as sensitivity analyses that consistently supported this result. The Office Director Review stated that although the findings were not independently reviewed by FDA, they were supportive of efficacy in later-onset SMA patients. ${ }^{7\left(\mathrm{p}^{4-5)}\right.}$ The Office Director Review also found results from 4 open-label trials conducted in SMA patients to be consistent with a beneficial effect of nusinersen in later-onset SMA patients, though the uncontrolled nature of the trials limited their interpretability. ${ }^{7(\mathrm{p} 5)}$

xvi. Ventavis (iloprost). ${ }^{8}$ Note that these studies are statistically positive in their primary hypotheses yet not exceptionally statistically persuasive.

\section{References}

1. FDA. Guidance for industry, providing clinical evidence of effectiveness for human drug and biological products, May 1998. https://www.fda.gov/media/71655/download.

2. FDA. Draft guidance for industry, providing clinical evidence of effectiveness for human drug and biological products, March 1997. 
3. Giapreza (angiotensin II), Cross-Discipline Team Leader Review Addendum, NDA 209360 (December 21, 2017).

4. Giapreza (angiotensin II), Office of Drug Evaluation 1: Decisional Memorandum, NDA 209360 (December 21, 2017).

5. Bevyxxa (betrixaban), Summary Review for Regulatory Action, NDA 208383 (June 23, 2017).

6. Gibson CM, Goldhaber SZ, Cohen AT, et al. When academic research organizations and clinical research organizations disagree: Processes to minimize discrepancies prior to unblinding of randomized trials. Am Heart J. 2017;189: 1-8.

7. Spinraza (nusinersen), Office of Drug Evalution-1: Decisional Memorandum, NDA 209531 (December 23, 2016).

8. Ventavis (iloprost), Office of Drug Evaluation I, Director Memorandum to File, NDA 21779 (December 28, 2004).

9. Peck CC, Wechsler J. Report of a workshop on confirmatory evidence to support a single clinical trial as a basis for new drug approval. Therapeutic Innovation \& Regulatory Science. 2002;36:517-34. 